University of Bergen, Department of Physics

Scientific/Technical Report No. 2000-05

ISSN 0803-2696

hep-th/0010176

October 2000

\title{
Critical Phenomenon of a Consistent $q$-Deformed Squeezed State
}

\author{
P. Osland ${ }^{a, *}$ and Jian-zu Zhang ${ }^{a, b, \#}$ \\ ${ }^{a}$ Department of Physics, University of Bergen, N-5007 Bergen, Norway \\ ${ }^{b}$ Institute for Theoretical Physics, Box 316, East China University of Science and \\ Technology, Shanghai 200237, P. R. China
}

\begin{abstract}
Within a self-consistent framework of $q$-deformed Heisenberg algebra and its equivalent framework of $q$-deformed boson commutation relations, which relate to the under-cutting phenomenon of Heisenberg's minimal uncertainty relation, special $q$-deformed squeezed states are constructed. Besides the similar local maximum squeezing as the one in the undeformed case, new strong squeezing appears when the amplitude of the related coherent state increases to large values. A critical phenomenon appears at a large value of the amplitude: the variance of one component of the quadrature of the light field approaches zero, but the variance of the corresponding conjugate quantity remains finite, which is a surprising deviation from Heisenberg's uncertainty relation. The qualitative character exposed by this $q$-squeezed state may provide some evidence about $q$-deformed effects in current experiments.
\end{abstract}

* E-mail address: Per.Osland@fi.uib.no

\# E-mail address: jzzhangw@online.sh.cn 


\section{Introduction}

In discussions of the Heisenberg minimal uncertainty relation special attention has been focused on coherent states and squeezed states of the light field [1]. The idea of squeezing has both fundamental and practical interests. A special physical proposal for obtaining squeezed state is based on the superposition of coherent states along a straight line on the plane of $\alpha$, the eigenvalues of the annihilation operators [2], which is easy to generalize to the $q$-deformed case. Heisenberg's uncertainty relation is a direct result of the Heisenberg commutation relation, which is the basis of quantum mechanics.

According to present tests of quantum electrodynamics, quantum theories based on Heisenberg's commutation relation are correct at least down to $10^{-18} \mathrm{~cm}$. The question arises whether there is a possible generalization of Heisenberg's commutation relation at shorter distances. In searching for such a possibility at short distances considerations of the space structure is a useful guide. If the space structure at such short distances exhibits a non-commutative property, and thus is governed by a quantum group symmetry, it has been shown that $q$-deformed quantum mechanics is a candidate for a possible pre-quantum theory at short distances. In the literature different frameworks of $q$-deformed quantum mechanics were established [2]18].

At the level of the uncertainty relation there are two kinds of modifications of Heisenberg's uncertainty relation [3, 9, 10, 16, 17, 18], implying under- or over-cutting of Heisenberg's minimal uncertainty relation. Because the framework of $q$-deformed Heisenberg algebra developed in Refs. [3, 7], which is associated with the under-cutting phenomenon [9, 10] shows clear physical contents: its relation to the corresponding $q$-deformed boson commutation relation and the limiting process of the $q$-deformed harmonic oscillator to the undeformed one are clear, in this paper our attention is focused on this framework. We investigate a special $q$-deformed squeezed state, which is a $q$-deformation of the squeezed state proposed in Ref. [2].

First we need to clarify consistent representations of $q$-occupation number states and

$q$-coherent states, which are consistent with the $q$-deformed Heisenberg algebra. Then we construct $q$-generalization of the squeezed states proposed in [2]. 
For such a $q$-deformed squeezed state there is a similar local maximum of squeezing as in the undeformed case, but the state shows an interesting new characteristic: As the amplitude of the related coherent state increases beyond the value corresponding to the local maximum of squeezing, the variance of one quadrature component of the light field shows a clear tendency of a second squeezing, whereas the corresponding variance for the undeformed case increases to the Heisenberg minimal uncertainty. Furthermore, the $q$ deformed one exhibits a critical phenomenon: when the amplitude of the related coherent state increases to a critical point the variance of one component of the quadrature of the light field approaches zero; meanwhile the variance of the corresponding conjugate operator remains finite. This is a surprising deviation from Heisenberg's uncertainty relation. The qualitative character exposed by this $q$-squeezed state is a clear indication of deviation from the Heisenberg uncertainty relation and may provide some evidence about $q$-deformed effects to present experiments.

In order to demonstrate the critical phenomenon of $q$-deformed squeezed states, in the following we first review the necessary background of $q$-deformed quantum mechanics.

\section{The $q$-deformed Heisenberg Algebra}

In terms of $q$-deformed phase space variables — the position operator $X$ and the momentum operator $P$, the following $q$-deformed Heisenberg algebra is developed [3, []:

$$
q^{1 / 2} X P-q^{-1 / 2} P X=i U, \quad U X=q^{-1} X U, \quad U P=q P U
$$

where $X$ and $P$ are hermitian and $U$ is unitary: $X^{\dagger}=X, P^{\dagger}=P, U^{\dagger}=U^{-1}$. In (2.1) the parameter $q$ is a fixed real number. It is important to make distinctions for different realizations of the $q$-algebra by different ranges of $q$ values [19 21]. Following Refs. [3, 7] we only consider the case $q>1$ in this paper. The operator $U$ is called the scaling operator, it closely relates to properties of the dynamics and plays an essential role in $q$-deformed quantum mechanics. The definition of the algebra (2.1) is based on the definition of the hermitian momentum operator $P$. However, if $X$ is assumed to be a hermitian operator in a Hilbert space the usual quantization rule $P \rightarrow-i \partial_{X}$ does not yield a hermitian momentum operator. Ref. [3] showed that a hermitian momentum operator $P$ is related to $\partial_{X}$ and $X$ 
in a nonlinear way by introducing a scaling operator $U$

$$
U^{-1}=q^{1 / 2}\left[1+(q-1) X \partial_{X}\right], \quad \bar{\partial}_{X}=-q^{-1 / 2} U \partial_{X}, \quad P=-\frac{i}{2}\left(\partial_{X}-\bar{\partial}_{X}\right) .
$$

Such defined hermitian momentum $P$ leads to $q$-deformed effects, which are exhibited

by the dynamical equation. For example, the perturbative expansion of the $q$-deformed Schrödinger equation possesses a complex structure, which amounts to some additional momentum-dependent interaction [3 5, [7], 99 11]. The nontrivial properties of $U$ imply that the algebra (2.1) has a richer structure than the Heisenberg commutation relation. In the limit $q \rightarrow 1^{+}$the scaling operator $U$ reduces to the unit operator, thus the algebra (2.1) reduces to the Heisenberg commutation relation.

\section{The $q$-deformed Boson Commutation Relation. Wess' Ansatz}

Now we consider the general framework of $q$-deformed boson commutation relations determined by the properties of the $q$-deformed annihilation, creation and number operators: $a, a^{\dagger}$ and $N$. The expressions for $a$ and $a^{\dagger}$ in terms of the $q$-deformed variables $X, P$, and the scaling operator $U$ (Wess' Ansatz) are [6]

$$
a=\eta U^{-2 M}+\beta U^{-M} P, \quad a^{\dagger}=\eta^{*} U^{2 M}+\beta^{*} P U^{M}
$$

where $M=1,2,3, \ldots$, and $\eta^{*}$ and $\beta^{*}$ are complex conjugates of the parameters $\eta$ and $\beta$. From Wess' Ansatz (3.1) it follows that $a$ and $a^{\dagger}$ satisfy the following $q$-deformed boson commutation relation:

$$
a a^{\dagger}-q^{-2 M} a^{\dagger} a=\left(1-q^{-2 M}\right) \eta \eta^{*}=1 .
$$

The right-hand side of (3.2) can be normalized to 1 for the case $q>1$, which determines $\eta$ up to a phase: $\eta=e^{i \phi} /\left(1-q^{-2 M}\right)^{1 / 2}$, where $\phi$ is a real number.

Wess' Ansatz (3.1) is determined by the requirement of equivalence of the algebras (2.1) and (3.1). The operators $a$ and $a^{\dagger}$ are related to the operator $X$ in a complicated way. (In (3.1) $X$ is nonlinearly included in the operator $U$.) It is interesting to note that in the limiting case $q \rightarrow 1^{+}$the $q$-deformed annihilation operator $a$ reduces to the undeformed 
one [6]. The $q$-deformed phase space variables $X, P$ and the scaling operator $U$ can be realized by the variables $\hat{x}$ and $\hat{p}$, which satisfy $\hat{x}=\hat{x}^{\dagger}, \hat{p}=\hat{p}^{\dagger}$ and $[\hat{x}, \hat{p}]=i$, as follows [3]:

$$
X=\frac{\left[\hat{z}+\frac{1}{2}\right]}{\hat{z}+\frac{1}{2}} \hat{x}, \quad P=\hat{p}, \quad U=q^{\hat{z}},
$$

where $\hat{z}=-i(\hat{x} \hat{p}+\hat{p} \hat{x}) / 2$, and $[A]$ is called the $q$-deformation of $A$, defined by $[A]=$ $\left(q^{A}-q^{-A}\right) /\left(q-q^{-1}\right)$. From (3.3) it follows that $X, P$ and $U$ satisfy (2.1). Furthermore, $\hat{x}$ and $\hat{p}$ are realized by conventional variables $x$ and $p$,

$$
\hat{x}=x, \quad \hat{p}=p+\gamma\left(1-q^{-2 M}\right)^{-1 / 2}, \quad p=-i \partial_{x},
$$

where $\gamma$ is a real parameter.

In order to study the behavior of $a$ in the limit $q \rightarrow 1^{+}$, let $q=e^{f}$, where $0<f \ll 1$. In the limit $q \rightarrow 1^{+}\left(f \rightarrow 0^{+}\right)$there are singular factors in $\eta$ and $\hat{p}$. The condition of cancellation of these two singular terms is $\beta \gamma=-e^{i \phi}$. If we take $e^{i \phi}=\mp i, \gamma= \pm(2 \omega)^{1 / 2}$, $\beta=i(2 \omega)^{-1 / 2}$, where the constant $\omega$ is the frequency of the ordinary oscillator, then in the limit $f \rightarrow 0^{+}$the $q$-deformed annihilation operator $a$ reduces to the ordinary annihilation operator: $a \rightarrow a_{0}=(\omega / 2)^{1 / 2} x+i(2 \omega)^{-1 / 2} p$. The $q$-deformed harmonic oscillators were first studied by Macfarlane [13] and Biedenharn [14.

The definition of the $q$-deformed number operator $N$ is the same as for the undeformed one: $[N, a]=-a,\left[N, a^{\dagger}\right]=a^{\dagger}$. But the relations between $a^{\dagger} a\left(a a^{\dagger}\right)$ and $N$ are involved. From (3.2) it follows that (in the following we only consider the case $M=1$ )

$$
a^{\dagger} a=q^{-(N-1)}[N], \quad a a^{\dagger}=q^{-N}[N+1],
$$

The eigenstate $|n\rangle_{q}$ of $N$ satisfies $N|n\rangle_{q}=n|n\rangle_{q}$, with ${ }_{q}\langle n \mid m\rangle_{q}=\delta_{n m}$ and

$$
a|n\rangle_{q}=q^{-(n-1) / 2}[n]^{1 / 2}|n-1\rangle_{q}, \quad a^{\dagger}|n\rangle_{q}=q^{-n / 2}[n+1]^{1 / 2}|n+1\rangle_{q} .
$$

The $q$-deformed vacuum $|0\rangle_{q}$ satisfies $a|0\rangle_{q}=0$.

We emphasize that the $q$-exponential factors in (3.5) and (3.6) are delicate points in applications of $q$-deformed oscillators. From (3.6) it follows that the $q$-occupation-number state is

$$
|n\rangle_{q}=\frac{q^{n(n-1) / 4}}{\sqrt{[n] !}}\left(a^{\dagger}\right)^{n}|0\rangle_{q}
$$


where $[n] !=[1][2] \cdots[n]$, and $[0] \equiv 1$.

As an application of the $q$-occupation number state (3.7) we consider the eigenvalues of the $q$-deformed Hamiltonian. In the literature there are two $q$-deformed Hamiltonians [3, 6, 10, 12]: $H_{\omega}=\omega\left(a^{\dagger} a+\frac{1}{2}\right)$ and $H_{Q, K}=\frac{1}{2} K^{2}+\frac{1}{2} \omega^{2} Q^{2}$, where $Q$ and $K$ are a pair of quadratures of $a$ and $a^{\dagger}$ :

$$
Q=\sqrt{\frac{1}{2 \omega}}\left(a+a^{\dagger}\right), \quad K=i \sqrt{\frac{\omega}{2}}\left(a^{\dagger}-a\right) .
$$

Refs. [3, 6, 10, 12] note the difference between $H_{\omega}$ and $H_{Q, K}$ that $H_{\omega}$ possesses conventional physical properties, but $H_{Q, K}$ probably does not permit a consistent physical interpretation. Thus we only consider the eigenvalues of $H_{\omega}$. From (3.5) it follows that $|n\rangle_{q}$ is an eigenstate of $H_{\omega}$ with eigenvalue

$$
E_{\omega, n}=\omega\left(q^{-(n-1)}[n]+\frac{1}{2}\right)=\omega\left[\left(1-q^{-2 n}\right) /\left(1-q^{-2}\right)+\frac{1}{2}\right]
$$

In the limit $q \rightarrow 1^{+}$, the spectrum $E_{\omega, n}$ in (3.9) is reduced to the undeformed one $E_{\omega, n}=$

$\omega\left(n+\frac{1}{2}\right)$. We also note that in the limit $n \rightarrow \infty$ the eigenvalues $E_{\omega, n}$ are bounded from above, $E_{\omega, \infty}=\omega\left[\left(1-q^{-2}\right)^{-1}+\frac{1}{2}\right]$. The spectrum $E_{\omega, n}$ in (3.9) is identified with the bounded spectrum of Ref. [6].

\section{Undercutting of Heisenberg's Minimal Uncertainty Relation}

The $q$-deformed uncertainty relation derived from the $q$-deformed boson commutation relations (3.2) differs qualitatively from that of Heisenberg [9, 10. In order to expose such characteristic quantum behavior governed by (3.2) we proceed as follows. From (3.2) (with $M=1$ ) and (3.8) it follows that the commutation relation between $Q$ and $K$ is:

$$
[Q, K]=i C, \quad C=1-\left(1-q^{-2}\right) a^{\dagger} a
$$

It was shown that in any state the expectation value of the operator $C$ satisfies [9, 10]

$$
0 \leq\langle C\rangle \leq 1
$$


In (4.2) the situation $\langle C\rangle=1$ only occurs for the case $q=1$, which means that Heisenberg's minimal uncertainty relation $\Delta Q \Delta K=\frac{1}{2}$ is undercut for $q>1$, where $\Delta A \equiv\left\langle(A-\bar{A})^{2}\right\rangle^{1 / 2}$, and $\bar{A} \equiv\langle A\rangle$. It is quite interesting to note that the possibility $\langle C\rangle=0$ can occur. In this case the $q$-deformed uncertainty relation differs essentially from that of Heisenberg. It means that there are special states, in which $\Delta Q$ and $\Delta K$ can be simultaneously zero. Within the $q$-deformed coherent state, such an example has been found [10, 17].

\section{5 q-deformed Coherent State [12]}

Using (3.2), (3.5)-(3.7) we construct $q$-coherent states, which are defined as eigenstates of the $q$-annihilation operator $a, a|\alpha\rangle_{q}=\alpha|\alpha\rangle_{q}$ with a complex eigenvalue $\alpha$. From the $q$-deformed boson commutation relations (3.2) it follows that the $q$-coherent state $|\alpha\rangle_{q}$ is represented as

$$
|\alpha\rangle_{q}=N_{\alpha}\left[|0\rangle_{q}+\sum_{n=1}^{\infty} \frac{q^{n(n-1) / 4} \alpha^{n}}{\sqrt{[n] !}}|n\rangle_{q}\right]=N_{\alpha} e_{q}\left(\alpha a^{\dagger}\right)|0\rangle_{q}
$$

where the function $e_{q}(x)$ is the $q$-deformed exponential function, which is defined as

$$
e_{q}(x)=1+\sum_{n=1}^{\infty} \frac{q^{n(n-1) / 2}}{[n] !} x^{n}
$$

When $q \rightarrow 1^{+}, e_{q}(x) \rightarrow \exp (x)$. We emphasize again that the $q$-exponential factors in (3.5)-(3.7), (5.1) and (5.2) guarantee the consistency of the theory. Note that there is an overlap between $|\alpha\rangle_{q}$ and $|-\alpha\rangle_{q}:{ }_{q}\langle\alpha \mid-\alpha\rangle_{q}=N_{-\alpha}^{*} N_{\alpha} e_{q}\left(-\alpha^{2}\right)$. For simplicity we may choose the phase factor so that $\alpha$ is real, thus in (5.1) the normalization constant is $N_{\alpha}=\left[e_{q}\left(\alpha^{2}\right)\right]^{-1 / 2}$.

\section{Critical Phenomenon of $q$-deformed Squeezed State}

We now consider a special $q$-squeezed state, which is a $q$-deformation of the squeezed state considered in Ref. [2]. An effective squeezing can be achieved by superposition of coherent states along a straight line on the $\alpha$ plane. For a single mode of frequency $\omega$ the electric field operator $E(t)$ is represented as $E(t)=E_{0}\left[a \exp (-i \omega t)+a^{\dagger} \exp (i \omega t)\right]$, where $a$ and $a^{\dagger}$ 
are the annihilation and creation operators of photon field. This squeezed state is defined as

$$
|\alpha, \pm\rangle_{q}=c_{ \pm}\left(|\alpha\rangle_{q} \pm|-\alpha\rangle_{q}\right)
$$

which satisfies $a|\alpha, \pm\rangle_{q}=\alpha c_{ \pm} c_{\mp}^{-1}|\alpha, \mp\rangle_{q}, a^{2}|\alpha, \pm\rangle_{q}=\alpha^{2}|\alpha, \pm\rangle_{q}$, and ${ }_{q}\langle\alpha, \pm \mid \alpha, \mp\rangle_{q}=0$. The normalization constants are $c_{ \pm}^{2}=e_{q}\left(\alpha^{2}\right) / 2\left[e_{q}\left(\alpha^{2}\right) \pm e_{q}\left(-\alpha^{2}\right)\right]$.

In the state $|\alpha,+\rangle_{q}$ squeezing appears. Let $a_{1}$ and $a_{2}$ be the dimensionless hermitian quadratures of the annihilation operator: $a=a_{1}+i a_{2}$. The variances of $a_{1}$ and $a_{2}$ in this state are:

$$
\begin{aligned}
& \left(\Delta a_{1}\right)^{2}=\frac{1}{4}+\frac{\alpha^{2}}{4}\left[2+\left(1+q^{-2}\right) \frac{e_{q}\left(\alpha^{2}\right)-e_{q}\left(-\alpha^{2}\right)}{e_{q}\left(\alpha^{2}\right)+e_{q}\left(-\alpha^{2}\right)}\right], \\
& \left(\Delta a_{2}\right)^{2}=\frac{1}{4}-\frac{\alpha^{2}}{4}\left[2-\left(1+q^{-2}\right) \frac{e_{q}\left(\alpha^{2}\right)-e_{q}\left(-\alpha^{2}\right)}{e_{q}\left(\alpha^{2}\right)+e_{q}\left(-\alpha^{2}\right)}\right] .
\end{aligned}
$$

The state $|\alpha,+\rangle_{q}$ is squeezed, i.e., the variance $\left(\Delta a_{2}\right)^{2}$ is less than Heisenberg's minimal uncertainty 0.25 . When $q \rightarrow 1^{+},\left(\Delta a_{2}\right)^{2}$ reduces to the undeformed value [2]

$$
\left(\Delta a_{2}\right)_{\mathrm{un}}^{2}=\frac{1}{4}-\frac{\alpha^{2}}{1+\exp \left(2 \alpha^{2}\right)} .
$$

For $\left(\Delta a_{2}\right)_{\text {un }}^{2}$, there is only one maximum squeezing, which appears at $\alpha_{\text {un }}^{2}=0.64$, where $\left(\Delta a_{2}\right)_{\text {un }}^{2}=0.111$. Beyond $\alpha_{\text {un }}^{2}$, as $\alpha^{2}$ increases, $\left(\Delta a_{2}\right)_{\text {un }}^{2}$ monotonically increases to Heisenberg's minimal uncertainty 0.25 .

For the $q$-deformed case, like the undeformed case, there is a similar local maximum squeezing. We consider a few numerical examples: $f=0.0001,0.001$ and 0.01; the corresponding values for $\left(\Delta a_{2}\right)^{2}$ are shown in Fig. 1. The local maximum squeezing $\left(\Delta a_{2}\right)^{2} \simeq 0.11$ appears at $\alpha_{\mathrm{q}-\mathrm{def}}^{2} \simeq 0.64$.

But (6.3) shows an essential new characteristic. In order to expose such new characteristic we proceed as follows.

The behavior of $\Delta a_{2}$ depends on the value of $q$. In the limit $q \rightarrow 1^{+}\left(f \rightarrow 0^{+}\right)$this behaviour can be studied analytically. Since the series expansion for the $q$-exponential, Eq. (5.2), is absolutely convergent, we may differentiate term by term, and find

$$
e_{q}(x) \simeq\left[1+\frac{1}{2}(q-1) x^{2}\right] e^{x}, \quad \text { as } q \rightarrow 1^{+}
$$




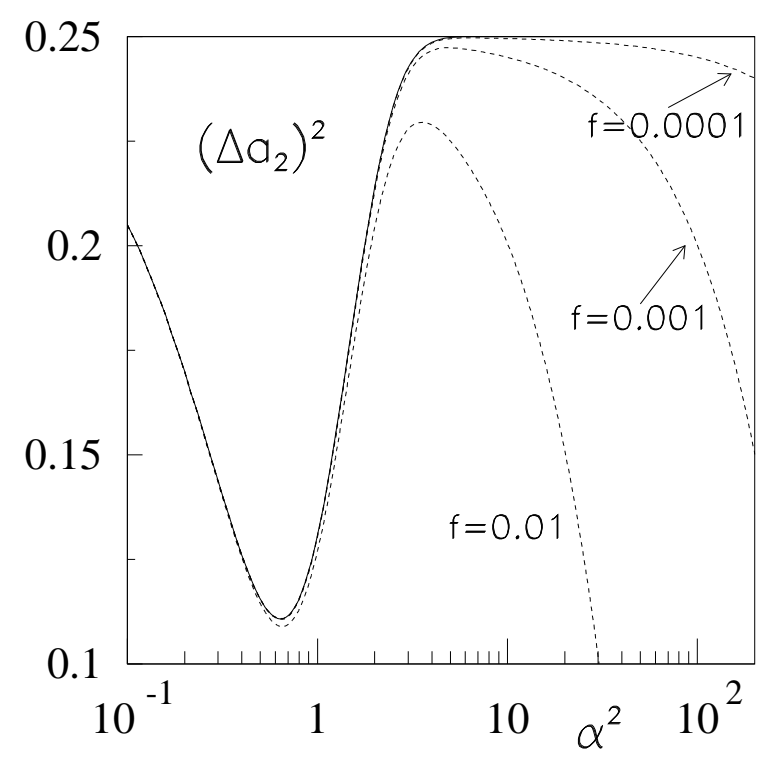

Figure 1: Variance $\left(\Delta a_{2}\right)^{2}$ vs. $\alpha^{2}$, for three values of $f$, where $q=e^{f}$ (dashed), and for the undeformed case (solid).

Substituting this into Eq. (6.3), we find

$$
\left(\Delta a_{2}\right)^{2} \simeq \frac{1}{4}-\frac{\alpha^{2}}{4}\left[2-\left(1+q^{-2}\right) \tanh \left(\alpha^{2}\right)\right] .
$$

For $q \rightarrow 1^{+}$, the critical value can be determined from Eq. (6.6).

For large $\alpha^{2}$ it follows that $\left(\Delta a_{2}\right)^{2}=\frac{1}{4}\left(1-2 \alpha^{2} f\right)$. Thus, the large $\alpha^{2}$-behavior of $\left(\Delta a_{2}\right)^{2}$ is quite different from that of the undeformed $\left(\Delta a_{2}\right)_{\mathrm{un}}^{2}$ : As $\alpha^{2}$ increases to large values, $\left(\Delta a_{2}\right)^{2}$ shows further strong squeezing; and at the point $\alpha_{c}^{2}=1 /(2 f)$ a critical squeezing appears: we obtain a zero variance $\left(\Delta a_{2}\right)^{2}=0$, meanwhile $\left(\Delta a_{1}\right)^{2}=1 /(2 f)$ remains finite. In Fig. 2 we show the $q$ - and $\alpha^{2}$-dependence of $\left(\Delta a_{2}\right)^{2}$ from Eq. (6.3). At low $f$, there is a local minimum around $\alpha^{2} \sim 0.64$, representing squeezing, followed by a plateau where $\left(\Delta a_{2}\right)^{2}$ approaches the Heisenberg value of 0.25 . Eventually, though, for yet larger values of $\alpha^{2}$, a second squeezing sets in, leading to a critical point where $\left(\Delta a_{2}\right)^{2}$ vanishes.

It should be emphasized that $q$-deformed quantum mechanics is essentially different from ordinary quantum mechanics. It shows qualitative deviations from Heisenberg's minimal uncertainty relation. For example, there are some special states, which permit simultaneously zero minimal uncertainties in a pair of conjugate operators [9, 10]. The 


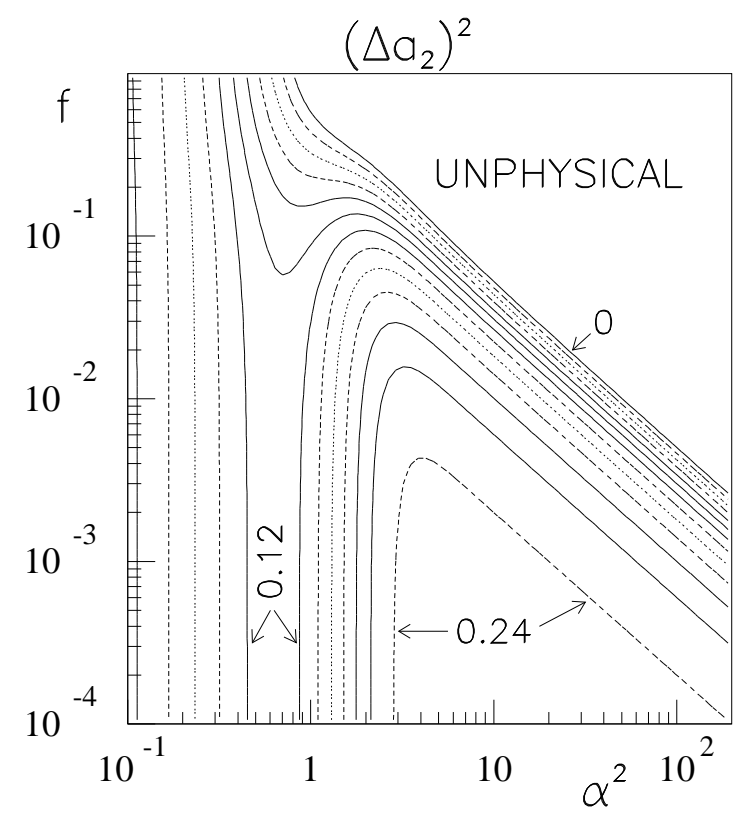

Figure 2: Variance $\left(\Delta a_{2}\right)^{2}$ vs. $\alpha^{2}$ and $f$. Contours of $\left(\Delta a_{2}\right)^{2}$ are drawn at values $0,0.02, \ldots, 0.24$. The region to the upper right is unphysical.

$q$-deformed coherent state [10, 17] is such an example. Here, we have discussed another case, the special $q$-squeezed state, which shows another qualitative deviation from Heisenberg's uncertainty relation. In a pair of conjugate operators, when one variance approaches zero, the other variance still remains finite, which is a surprising deviation from Heisenberg's uncertainty relation: for quantum mechanics if one variance approaches zero the variance of the conjugate operator should approach infinity.

It should be pointed out that if $q$-deformed quantum mechanics is a relevant physical theory, then its effects mainly manifest themselves at very short distances much smaller than $10^{-18} \mathrm{~cm}$; its correction to ordinary quantum mechanics must be very small in the energy range of present experiments, which means that the parameter $q$ must be very close to one. The critical phenomenon exposed in the $q$-squeezed state (6.1) gives an example, which is a clear indication of $q$-deformed effects and may provide some evidence about such effects to present experiments. The above analysis suggests that in experiments one should consider a light field with large $\alpha^{2}$ and examine the large- $\alpha^{2}$ behavior of the variance of the $a_{2}$ component. 
This work has been supported by the Research Council of Norway. JZZ would like to thank the Department of Physics, University of Bergen for hospitality. His work has also been supported by the National Natural Science Foundation of China, and by the Shanghai Education Development Foundation.

\section{References}

[1] D. F. Walls, Nature, 306 (1983) 141; R. E. Slusher, L. W. Hollberg, B. Yurke, J. C. Mertz and J. F. Valley, Phys. Rev. Lett. 55 (1985) 2409; L.-A. Wu, H. J. Kimble, J. L. Hall, and H. Wu, Phys. Rev. Lett. 57 (1986) 2520; H. J. Kimble and D. F. Walls, J. Opt. Soc. Am. B4 (1987) 1450; E. Giacobino and C. Fabre, Appl. Phys. B55 (1992) 189.

[2] J. Janszky and An. V. Vinogradov, Phys. Rev. Letters 64 (1990) 2771; and references therein.

[3] M. Fichtmüller, A. Lorek and J. Wess, Z. Phys. C 71 (1996) 533.

[4] J. Schwenk and J. Wess, Phys. Lett. B 291 (1992) 273.

[5] A. Lorek and J. Wess, Z. Phys. C 67 (1995) 671.

[6] A. Lorek, A. Ruffing and J. Wess, Z. Phys. C 74 (1997) 369.

[7] A. Hebecker, S. Schreckenberg, J. Schwenk, W. Weich and J. Wess, Z. Phys. C 64 (1994) 355.

[8] J. Wess and B. Zumino, Nucl. Phys. (Proc. Suppl.) 18B (1991) 302.

[9] Jian-zu Zhang, Phys. Lett. B440 (1998) 66.

[10] Jian-zu Zhang, Phys. Lett. A262 (1999) 125.

[11] Jian-zu Zhang, Chinese Phys. Lett. 17 (2000) 91; Phys. Lett. B477 (2000) 361.

[12] C.A. Nelson and M.H. Fields, Phys. Rev. A51 (1995) 2410. 
[13] A. J. Macfarlane, J. Phys. A 22 (1989) 4581.

[14] L.C. Biedenharn, J. Phys. A 22 (1989) L873.

[15] C.-P. Sun, and H.-C. Fu, J. Phys. A22 (1989) L983; M. Chaichian and P. Kulish, Phys. Lett. B234 (1990) 72; P.P. Kulish and E.V. Damashinsky, J. Phys. A23 (1990) L415; M. Arik and D. D. Coon, J. Math. Phys. 17 (1976) 524.

[16] S. Codriansky, Phys. Lett. A184 (1993) 381.

[17] R.J. McDermott and A.I. Solomon, J. Phys. A 27 (1994) L15.

[18] A. Kempf, J. Math. Phys. 35 (1994) 4483.

[19] C. Zachos, Contem. Math. 134 (1992) 351.

[20] C. A. Nelson and M. G. Gartley, J. Phys. A27 (1994) 3857.

[21] A. I. Solomon and J. Katriel, J. Phys. A26 (1993) 5443. 